\title{
Correction to: Predictive and prognostic clinical and pathological factors of nivolumab efficacy in non-small-cell lung cancer patients
}

\author{
J. Garde-Noguera' ${ }^{1}$ - P. Martin-Martorell ${ }^{2} \cdot$ M. De Julián ${ }^{3}$. J. Perez-Altozano ${ }^{4}$. C. Salvador-Coloma ${ }^{5}$. \\ J. García-Sanchez ${ }^{1}$ - A. Insa-Molla ${ }^{2} \cdot$ M. Martín $^{6} \cdot$ X. Mielgo-Rubio $^{7} \cdot$ S. Marin-Liebana ${ }^{8} \cdot$ A. Blasco-Cordellat ${ }^{9}$. \\ S. Blasco-Molla ${ }^{10} \cdot$ R. Gironés ${ }^{11}$. D. Marquez-Medina ${ }^{12} \cdot$ F. Aparisi ${ }^{13} \cdot$ M. C. Bas Cerda ${ }^{14} \cdot$ S. Macia-Escalante ${ }^{15}$. \\ A. Sánchez $z^{3} \cdot$ O. Juan-Vidal ${ }^{5}$
}

Published online: 9 July 2018

๑) Federación de Sociedades Españolas de Oncología (FESEO) 2018

\section{Correction to: Clinical and Translational Oncology https://doi.org/10.1007/s12094-017-1829-5}

Unfortunately some of the names of the authors were spelled incorrectly in the published original article. The correct names are:

Author 2: P. Martin-Martorell

Author 4: J. Perez-Altozano

Author 5: C. Salvador-Coloma
Author 6: J. García-Sanchez

Author 7: A. Insa-Molla

Author 9: X. Mielgo-Rubio

Author 10: S. Marin-Liebana

Author 11: A. Blasco-Cordellat

Author 12: S. Blasco-Molla

Author 14: D. Marquez-Medina

Author 17: S. Macia-Escalante

The original article can be found online at https://doi.org/10.1007/ s12094-017-1829-5.

J. Garde-Noguera

javiergardenoguera1@gmail.com

Hospital Arnau de Vilanova, C/Sant Climent, 12, 46015 Valencia, Spain

2 University Hospital Clínic de Valencia, Valencia, Spain

3 Hospital Provincial de Castellón, Castellón, Spain

4 Hospital General de Elche, Elche, Spain

5 Hospital Universitari I Politècnic La Fe, Valencia, Spain

6 Hospital Dr Peset, Valencia, Spain

7 Hospital Universitario Fundación Alcorcón, Alcorcón, Spain
Hospital de Manises, Manises, Spain

9 Hospital General Universitario de Valencia, Valencia, Spain

10 Hospital de Sagunto, Sagunto, Spain

11 Hospital Lluis Alcanyís, Xátiva, Spain

12 Hospital Arnau de Vilanova, Lleida, Spain

13 Hospital Virgen de los Lirios, Alcoy, Spain

14 Universidad Politecnica de Valencia, Valencia, Spain

15 Pivotal CRO, Madrid, Spain 